\title{
Nonlinear dynamic response of beam and its application in nanomechanical resonator
}

\author{
Yin Zhang • Yun Liu • Kevin D. Murphy
}

Received: 10 March 2011 / Revised: 13 April 2011 / Accepted: 7 June 2011

(C)The Chinese Society of Theoretical and Applied Mechanics and Springer-Verlag Berlin Heidelberg 2011

\begin{abstract}
Nonlinear dynamic response of nanomechanical resonator is of very important characteristics in its application. Two categories of the tension-dominant and curvaturedominant nonlinearities are analyzed. The dynamic nonlinearity of four beam structures of nanomechanical resonator is quantitatively studied via a dimensional analysis approach. The dimensional analysis shows that for the nanomechanical resonator of tension-dominant nonlinearity, its dynamic nonlinearity decreases monotonically with increasing axial loading and increases monotonically with the increasing aspect ratio of length to thickness; the dynamic nonlinearity can only result in the hardening effects. However, for the nanomechanical resonator of the curvature-dominant nonlinearity, its dynamic nonlinearity is only dependent on axial loading. Compared with the tension-dominant nonlinearity, the curvature-dominant nonlinearity increases monotonically with increasing axial loading; its dynamic nonlinearity
\end{abstract}

The project was supported by the National Natural Science Foundation of China (10721202 and 11023001) and the Chinese Academy of Sciences (KJCX2-EW-L03).

\author{
Y. Zhang (区) \\ State Key Laboratory of Nonlinear Mechanics, \\ Institute of Mechanics, Chinese Academy of Sciences, \\ 100190 Beijing, China \\ e-mail: zhangyin@lnm.imech.ac.cn \\ Y. Liu \\ Faculty of Information and Automation, \\ Kunming University of Science and Technology, \\ 650051 Kunming, China \\ K.D. Murphy \\ Department of Mechanical Engineering, \\ The University of Connecticut, \\ Storrs, CT 06269, USA
}

can result in both hardening and softening effects. The analysis on the dynamic nonlinearity can be very helpful to the tuning application of the nanomechanical resonator.

Keywords Resonator - Dynamic response - Dynamic nonlinearity $\cdot$ Dimensional analysis

\section{Introduction}

The fabrication of micro/nano-electromechanical systems (MEMS/NEMS) is made possible due to the advancing technologies and state-of-art in the fields of micro/nanometrescale processing and machining [1-18]. As its characteristic frequency scales upwards with decreasing size [9], micro/nanometre-scale mechanical resonator/oscillator with high and ultrahigh characteristic frequency is suitable for those applications requiring both high responsivity and high frequency operation [10], such as charge detection [2], ultra-sensitive mass sensing [7,12-17], signal processing/mixing [8] and possible quantum effect study on a macroscopic system [11]. Charles Babbage in 1834 designed the first mechanical analytic engine [5], which is viewed as the forerunner of modern computer [19]. The advent of electronic transistor and magnetic storage technologies in 1960s, which were much superior to the mechanism of mechanical moving element in both manipulation speed and data density, resulted in the abandonment of Babbage's idea of mechanical computer [19]. Recent breakthrough in the fabrication of NEMS resonator with the fundamental characteristic frequency of $1 \mathrm{GHz}$ and higher $[9,11]$ intrigues further research interest in this area because such breakthrough could eventually lead to the realization of high performance mechanical computer which can compete with current electronic computer. Mechanical resonator with $\mathrm{GHz}$ characteristic frequency which moves on time scale of a nanosecond or less makes such competition possible [19]. Recent suc- 
cessful fabrication of controllable nanomechanical memory element [5] and switch [6] demonstrates such rivalry potential. Even before the advent of $\mathrm{GHz}$ nanomechanical resonator, with the trends of the nanomechanical resonator with higher and higher fundamental characteristic frequency of $\mathrm{MHz}$ order being fabricated, Roukes called for the careful reexamination on the established dogma of digital electronic age and re-consideration for the abandoned design of mechanical computer envisaged by Charles Babbage [19].

With respect to the (promising) applications and advantages of nanomechanical resonator, it still faces many serious technical challenges and difficulties, such as the fluctuation due to thermomechanical noise $[10,20,21]$; low $Q$ factor with decreasing size $[10,17,18,21,22]$, increasing magnetic field [21], increasing ambient pressure [4,21] and dynamic nonlinear response [3-8,10,11,21,23,24]. Recent findings on the initial conditions-dependent antiresonance response of weakly nonlinear nanomechanical resonator [24] and strongly nonlinear response of doubly clamped nanomechanical resonator [10] demonstrate some application limitations of nanomechanical resonators because of nonlinearity. However, nonlinearity is not always harmful. The nonlinear dynamic response of nanomechanical resonator results in a bistable region, which has been successfully used as a mechanism to realize mechanical binary state $[5,6]$. The nonlinearity can also be utilized to improve the charge sensitivity [25] and as a frequency stablizer in a feedback loop [26].

Both the flexural vibration $[5,6,8,10,16,23,26]$ and torsional vibration $[13,14]$ of nanomechanical resonator are described by Duffing equation, which possesses cubic nonlinearity [27]. The cubic nonlinearity of nanomechanical resonator beam structure arises from two sources: (1) tension due to nonlinear mid-plane stretching [10] and (2) curvature nonlinearity [10,28]. For doubly clamped [10], doubly hinged and hinged-clamped beam structures [27], the tension due to the nonlinear mid-plane stretching is dominantly responsible for the cubic nonlinearity in Duffing equation. For a cantilever beam, curvature nonlinearity is dominant $[10,28]$. For doubly clamped, doubly hinged and hinged-clamped beam structures, their tensiondominant nonlinearity can only be valid when the structure is not slack. Slack here means that the structural length is (much) longer than the distance between the contacts/structure ends [4]. For a nanotube or a nanowire-based nanomechanical resonator which has large aspect ratio of length to thickness/radius $[4,10]$, the slack doubly clamped nanomechanical resonator should be modeled as catenary rather than beam [4]. For a catenary structure, it is curvaturedominant nonlinearity [4]. To the authors' best knowledge, the nonlinearity effects of Duffing-type nanomechanical resonators has not been comprehensively studied. Anderson et al. did both theoretical and experimental studies on the curvature-dominant nonlinear dynamic response of a cantilever beam [28]. However, the axial/longitudinal loading is not included as a parameter in their studies [28], which can significantly influence the nonlinearity of the nanomechanical resonator as shown in this paper. Tunability is a much sought-after capability in nanomechanical resonator applications [29] and it can be realized by changing the axial loading inside the nanomechanical resonator $[4,16,18]$. Therefore, it is necessary and important to incorporate the axial loading into the dynamic nonlinear response study of nanomechanical resonator. Postma et al. studied the critical amplitude for the tension-dominant nonlinearity onset of a doubly clamped nanomechanical resonator [10]. In Ref. [10], both the aspect ratio and the axial loading are considered as two important factors impacting the beam nonlinear dynamic response. However, only one type structure of the tension-dominant nonlinearity is analyzed in Ref. [10]; the detailed and comprehensive analyses on these two factors are not presented. Lifshitz and Cross [30] presented a very detailed analysis on the micro/nanomechanical resonators. However, our nondimensionalization scheme on the beam governing equation is different from Lifshitz and Cross's, which leads to a different Duffing equation.

Recent molecular dynamics simulation and initial experiments appear to indicate that the continuum mechanics model breaks down only for the structures with the cross section of the order of tens of lattice constants [17]. A continuum mechanics approach is thus taken in this paper. The analysis work of this paper consists of two parts. One part is to offer an overview of the dynamic nonlinear response of Duffing equation with different parameters. Duffing equation is for the system of single degree of freedom (DOF) and nanomechanical resonator is a continuous system of beam structure. The other part is to use modal analysis and Galerkin method to transform the differential-integral and differential governing equations into single DOF Duffing equation. Based on that, dimensional analysis is used to study the dynamic nonlinear response of nanomechanical resonator. Peano and Thorwart's study on the dynamic nonlinear response of nanomechanical resonator is to treat the "nonlinearity coefficient" as a variable parameter [23]. The dimensional analysis presented here relates the "nonlinearity coefficient" with the physical parameters and offers a guide on how to decrease or increase the dynamic nonlinear response of a nanomechanical resonator for different application purposes.

\section{Duffing equation}

Duffing equation of single DOF system is as follows [27]

$\frac{\mathrm{d}^{2} u}{\mathrm{~d} t^{2}}+2 \epsilon \mu \frac{\mathrm{d} u}{\mathrm{~d} t}+\omega_{\mathrm{o}}^{2} u+\epsilon \alpha u^{3}=\epsilon k \cos (\Omega t)$.

Here $u$ is the system displacement, $\omega_{0}$ is the system resonant frequency without damping, $\mu$ is positive damping coefficient, $\alpha$ can be either positive (hardening effects) or negative (softening effects), $\epsilon$ is a small parameter, $\epsilon k$ is the 
amplitude of external excitation and $\Omega$ is its driving frequency. Here the excitation is assumed independent of the state of system $\left(u, \mathrm{~d} u / \mathrm{d} t\right.$ and $\left.\mathrm{d}^{2} u / \mathrm{d} t^{2}\right)$, which is called ideal sources of energy [27]. If the excitation is dependent on the state of the system, it is called nonideal sources of energy. Some of nanomechanical resonators are driven electrostatically $[13,14,16]$ and the electrostatic force $f_{\mathrm{e}} \propto(d-u)^{-2}(d$ is the gap distance between the resonator and substrate) [31]. Therefore, electrostatic driving force is a nonideal source of energy. With the discretization of Galerkin method and proper truncation of the Taylor series, the electrostatically driven system can still be expressed similar to the abovementioned governing equation with cubic nonlinearity [31]. Physically, classifying these two cases is important because it indicates whether the excited system response influences the excitation source(s). For the system of ideal sources of energy, the excited system response influence on the excitation source is none or negligible [27]. Here the system primary response, i.e. $\Omega \approx \omega_{0}$ is studied and the parameter $\sigma$ named detuning is introduced. Detuning parameter $\sigma$ is to quantitatively describe the nearness of $\Omega$ to $\omega_{\mathrm{o}}$. Accordingly, we have

$\Omega=\omega_{\mathrm{o}}+\epsilon \sigma$,

where $\sigma=O(1)$. Here the multiscale perturbation method is applied to derive the dynamic nonlinear response of Duffing equation. We assume

$$
\begin{aligned}
& u(t ; \epsilon)=u_{\mathrm{o}}\left(T_{\mathrm{o}}, T_{1}\right)+\epsilon u_{1}\left(T_{\mathrm{o}}, T_{1}\right) \\
& +\cdots(\text { higher order terms), } \\
& T_{\mathrm{o}}=t, \quad T_{1}=\epsilon t,
\end{aligned}
$$

and

$$
\begin{aligned}
\frac{\mathrm{d}}{\mathrm{d} t} & =\frac{\mathrm{d} T_{\mathrm{o}}}{\mathrm{d} t} \frac{\partial}{\partial T_{\mathrm{o}}}+\frac{\mathrm{d} T_{1}}{\mathrm{~d} t} \frac{\partial}{\partial T_{1}}+\cdots \\
& =\mathrm{D}_{\mathrm{o}}+\epsilon \mathrm{D}_{1}+\cdots \text { (higher order terms) } \\
\frac{\mathrm{d}^{2}}{\mathrm{~d} t^{2}} & =\mathrm{D}_{\mathrm{o}}^{2}+2 \epsilon \mathrm{D}_{\mathrm{o}} \mathrm{D}_{1}+\cdots \text { (higher order terms). }
\end{aligned}
$$

In conjunction with Eqs. (3) and (4), Eq. (1) is now rewritten as the following form with the truncation of higher order terms

$$
\begin{gathered}
\left(\mathrm{D}_{\mathrm{o}}^{2}+2 \epsilon \mathrm{D}_{\mathrm{o}} \mathrm{D}_{1}\right)\left(u_{\mathrm{o}}+\epsilon u_{1}\right)+2 \epsilon \mu\left(\mathrm{D}_{\mathrm{o}}+\epsilon \mathrm{D}_{1}\right)\left(u_{\mathrm{o}}+\epsilon u_{1}\right) \\
+\omega_{\mathrm{o}}^{2}\left(u_{\mathrm{o}}+\epsilon u_{1}\right)+\epsilon \alpha\left(u_{\mathrm{o}}+\epsilon u_{1}\right)^{3}=\epsilon k \cos (\Omega t) .
\end{gathered}
$$

Equating the coefficients of $\epsilon^{0}$ and $\epsilon^{1}$ in Eq. (5), we obtain

$$
\begin{aligned}
\epsilon^{0}: \mathrm{D}_{\mathrm{o}}^{2} u_{\mathrm{o}}+\omega_{\mathrm{o}}^{2} u_{\mathrm{o}}= & 0, \\
\epsilon^{1}: \mathrm{D}_{\mathrm{o}}^{2} u_{1}+\omega_{\mathrm{o}}^{2} u_{1}= & -2 \mathrm{D}_{\mathrm{o}} \mathrm{D}_{1} u_{\mathrm{o}}-2 \mu \mathrm{D}_{\mathrm{o}} u_{\mathrm{o}} \\
& -\alpha u_{\mathrm{o}}^{3}+k \cos (\Omega t) .
\end{aligned}
$$

From Eq. (6), $u_{\mathrm{o}}$ is solved as follows

$u_{\mathrm{o}}=A\left(T_{1}\right) \cos \left[w_{\mathrm{o}} T_{\mathrm{o}}+B\left(T_{1}\right)\right]$.

Physically, $A\left(T_{1}\right)$ is the (unknown) system response ampli- tude and $B\left(T_{1}\right)$ is the (unknown) phase shift angle. Substituting $u_{\mathrm{o}}$ solution of Eq. (8) into Eq. (7), we have

$$
\begin{aligned}
\mathrm{D}_{\mathrm{o}}^{2} u_{1}+\omega_{\mathrm{o}}^{2} u_{1}= & 2 A^{\prime} \omega_{\mathrm{o}} \sin \left(\omega_{\mathrm{o}} T_{\mathrm{o}}+B\right) \\
& +2 A B^{\prime} \omega_{\mathrm{o}} \cos \left(\omega_{\mathrm{o}} T_{\mathrm{o}}+B\right) \\
& +2 A \mu \omega_{\mathrm{o}} \sin \left(\omega_{\mathrm{o}} T_{\mathrm{o}}+B\right) \\
& -\frac{\alpha^{3} A^{3}}{4}\left[3 \cos \left(\omega_{\mathrm{o}} T_{\mathrm{o}}+B\right)+\cos 3\left(\omega_{\mathrm{o}} T_{\mathrm{o}}+B\right)\right] \\
& +k\left[\cos \left(\sigma T_{1}-B\right) \cos \left(\omega_{\mathrm{o}} T_{\mathrm{o}}+B\right)\right. \\
& \left.-\sin \left(\sigma T_{1}-B\right) \sin \left(\omega_{\mathrm{o}} T_{\mathrm{o}}+B\right)\right] .
\end{aligned}
$$

Here $A^{\prime}=\mathrm{d} A / \mathrm{d} T_{1}$ and $B^{\prime}=\mathrm{d} B / \mathrm{d} T_{1}$. In Eq. (9), the following properties of triangular functions are used

$$
\begin{aligned}
& \cos (\Omega t)= \cos \left(\omega_{\mathrm{o}} T_{\mathrm{o}}+\sigma T_{1}\right) \\
&= \cos \left[\left(\omega_{\mathrm{o}} T_{\mathrm{o}}+B\right)+\left(\sigma T_{1}-B\right)\right] \\
&= \cos \left(\sigma T_{1}-B\right) \cos \left(\omega_{\mathrm{o}} T_{\mathrm{o}}+B\right) \\
&-\sin \left(\sigma T_{1}-B\right) \sin \left(\omega_{\mathrm{o}} T_{\mathrm{o}}+B\right), \\
& u_{\mathrm{o}}^{3}=A^{3} \cos ^{3}\left(\omega_{\mathrm{o}} T_{\mathrm{o}}+B\right) \\
&=\frac{3 \cos \left(\omega_{\mathrm{o}} T_{\mathrm{o}}+B\right)+\cos 3\left(\omega_{\mathrm{o}} T_{\mathrm{o}}+B\right)}{4} A^{3} .
\end{aligned}
$$

The secular terms of the particular solution of Eq. (9) will be eliminated if the coefficients of $\sin \left(\omega_{0} T_{0}+B\right)$ and $\cos \left(\omega_{\mathrm{o}} T_{\mathrm{o}}+B\right)$ terms are set to zero. Therefore, we have the following two equations

$\sin \left(\omega_{\mathrm{o}} T_{\mathrm{o}}+B\right): 2 A^{\prime} \omega_{\mathrm{o}}+2 A \mu \omega_{\mathrm{o}}-k \sin \gamma=0$,

$\cos \left(\omega_{\mathrm{o}} T_{\mathrm{o}}+B\right): 2 A\left(\sigma-\gamma^{\prime}\right) \omega_{\mathrm{o}}-\frac{3 \alpha A^{3}}{4}+k \cos \gamma=0$.

Here $\gamma=\sigma T_{1}-B$ and $\gamma^{\prime}=\mathrm{d} \gamma / \mathrm{d} T_{1}$. Steady state motion occurs when $A^{\prime}=\gamma^{\prime}=0$ [27]. Therefore, Eqs. (12) and (13) correspondingly change to the following equations for steady state motion

$\mu=\frac{k}{2 \omega_{0} A} \sin \gamma$,

$\sigma-\frac{3 \alpha A^{2}}{8 \omega_{0}}=-\frac{k}{2 \omega_{0} A} \cos \gamma$

Once again, by using the property of triangular function, we derive the following equation from Eqs. (14) and (15)

$\mu^{2}+\left(\sigma-\frac{3 \alpha A^{2}}{8 \omega_{0}}\right)^{2}=\frac{k^{2}}{4 \omega_{\mathrm{o}}^{2} A^{2}}$.

Equation (16) is called frequency-response equation, which is the same as that derived via a complex variable approach [27]. Equation (16) can be re-written as the following one

$\sigma=\frac{3 \alpha A^{2}}{8 \omega_{\mathrm{o}}} \pm \sqrt{\frac{k^{2}}{4 \omega_{\mathrm{o}}^{2} A^{2}}-\mu^{2}}$. 
In Nayfeh and Mook's book [27], $\alpha$ is treated as the parameter indicating the nonlinearity of dynamic response of Duffing equation. The system response is linear $(\alpha=0)$ or nonlinear $(\alpha \neq 0)$. While, as shown later in the analysis for the nanomechanical resonator, the characteristic frequency $\omega_{\mathrm{o}}$ varies with the axial loading. It is more proper here to use parameter $\alpha / \omega_{0}$ instead of $\alpha$ as the parameter indicating the system effective nonlinearity. Similarly, we use $k / \omega_{0}$ instead of $k$ to indicate the effective excitation amplitude on the system response. The parabola of $\sigma=3 \alpha A^{2} /\left(8 \omega_{0}\right)$ is often called backbone curve [27]. Indicated by Eq. (16) or (17), when the external driving frequency varies as $\Omega=\omega_{\mathrm{o}}+\epsilon \sigma$, $\alpha / \omega_{\mathrm{o}}$ (equivalent to the nonlinearity coefficient in Peano and Thorwart's study [23]), $k / \omega_{\mathrm{o}}$ (effective external driving amplitude) and $\mu$ (damping coefficient) are the three factors determining the system steady-state response. In nanomechanical resonator application, $k / \omega_{\mathrm{o}}$ is a control parameter and $\mu$ is fixed with given material properties, structure dimensions, related ambient conditions [21] and axial loading [18]. Here the analysis focuses only on the nonlinearity coefficient of $\alpha / \omega_{\mathrm{o}}$ as a function of structure dimensions and axial loading.

Figure 1 shows the system frequency response for $k / \omega_{\mathrm{o}}=0.05$ and $\mu=0.1$ when $\alpha / \omega_{\mathrm{o}}$ varies as $-40,-20$ (softening effects), 0 (linear response) and 20, 40 (hardening effects). The dashed lines stand for backbone curves. In Fig. 1, the magnitude of parameter $\alpha / \omega_{\mathrm{o}}$ or backbone curve determines how the whole system response curve bends, which reflects the nonlinearity. Figure 2 shows the system frequency response for $\alpha / \omega_{\mathrm{o}}=40$ and $\mu=0.1$ when $k / \omega_{\mathrm{o}}$ varies as $0.01,0.02,0.03,0.04$ and 0.05 . All these five response curves share one backbone curve and $k / \omega_{0}$ behaves as an amplification factor to amplify the nonlinear response along the backbone curve. Figure 3 shows the system frequency response with $\alpha / \omega_{0}=40$ and $k / \omega_{0}=0.05$ when $\mu=0.2,0.1$ and 0.05. Similar to those in Fig. 2, Fig. 3 shows that all three curves share one backbone curve and $\mu^{-1}$ behaves as an amplification factor. Figure 4 shows the jump and hysteresis phenomenon of a hardening Duffing oscillator with $\alpha / \omega_{0}=40, k / \omega_{0}=0.05$ and $\mu=0.1$. As $\sigma$ increases to point $J_{1}$, the system response becomes unstable and will experience a subsequent jump to point $J_{2}$ if further increasing $\sigma$. After the response jumps to lower branch, if decreasing $\sigma$, the response will keep stable until point $J_{3}$ and will jump to point $J_{4}$. As mentioned before in Sect. 1, the jump and hysteresis between the bistable region as shown in Fig. 4 are utilized as a mechanism to realize a mechanical binary state $[5,6]$.

The Duffing equation form of Eq. (1) and the related perturbation solution procedures above implicitly assume a small damping. The quality factor $Q\left(Q \propto(\epsilon \mu)^{-1}\right)$ of nanomechanical resonator shows very strong size-dependent property that $Q$ factor reduces with the decrease of the resonator size $[10,17,18,21,22]$. Equation (17) obtained by the perturbation method will become inaccurate when $Q$ factor

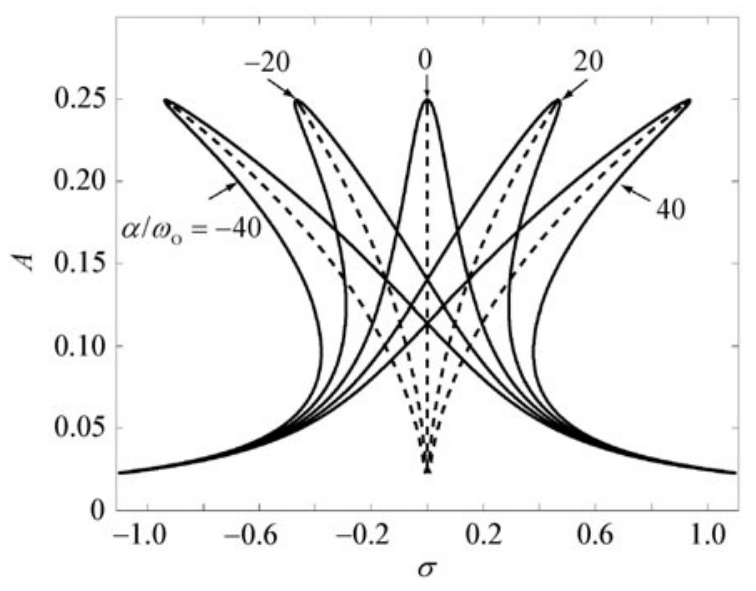

Fig. 1 The nonlinearity effects study of the steady state dynamic response of Duffing equation. $k / \omega_{0}=0.05$ and $\mu=0.1$ are fixed. The dashed lines stand for backbone curves

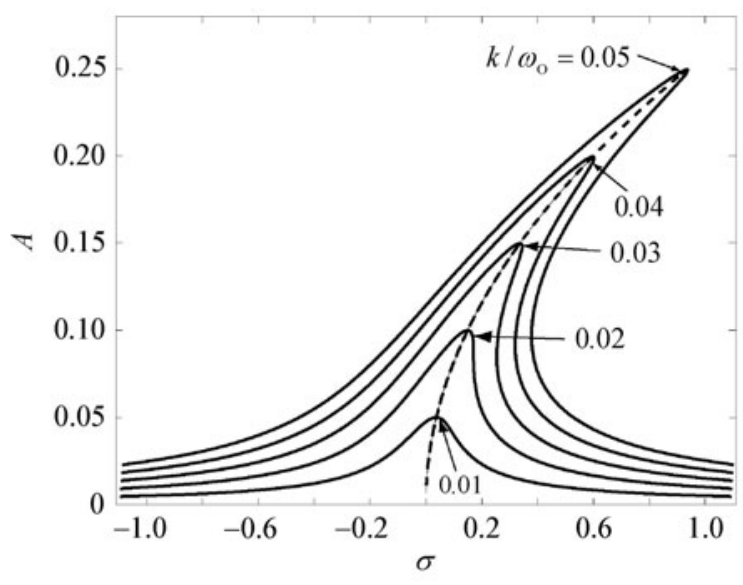

Fig. 2 The excitation amplitude study of the steady state dynamic response of Duffing equation. $\alpha / \omega_{\mathrm{o}}=40$ and $\mu=0.1$ are fixed, $k / \omega_{0}$ varies as $0.01,0.02,0.03,0.04$ and 0.05

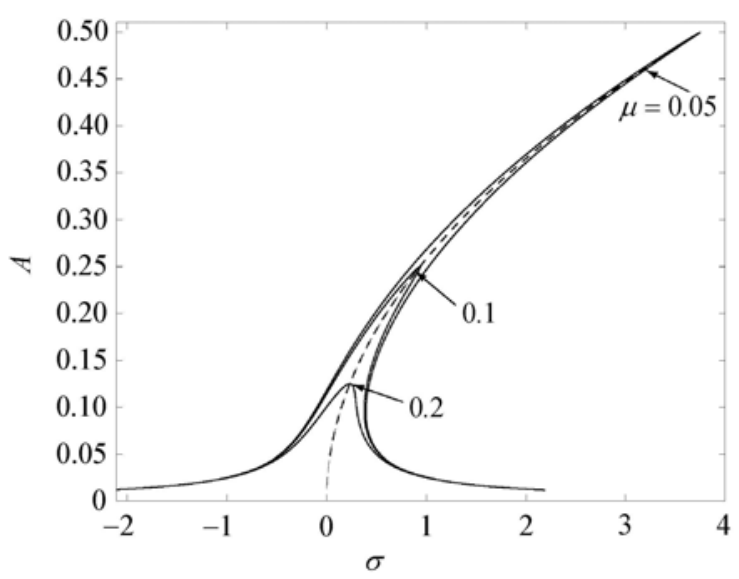

Fig. 3 The quality factor/damping study of the steady state dynamic response of Duffing equation. $\alpha / \omega_{\mathrm{o}}=40$ and $k / \omega_{\mathrm{o}}=0.05$ are fixed, $\mu$ varies as $0.2,0.1$ and 0.05 


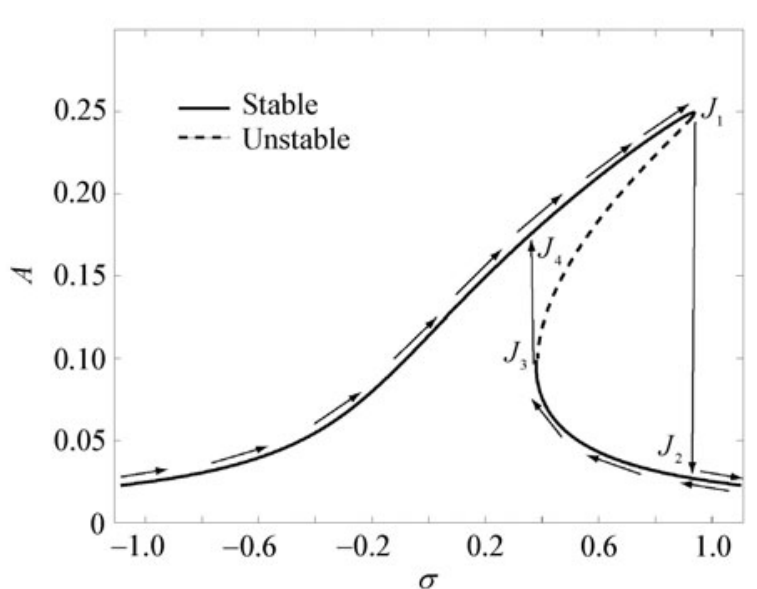

Fig. 4 Jump and hysteresis phenomenon in steady state response of Duffing equation

is very small. In Figs. 1 to 4 , the damping coefficient $\mu$ and the nonlinearity parameter $\alpha / \omega_{0}$ are in fact treated as two independent parameters. As demonstrated later, $\alpha / \omega_{\mathrm{o}}$ of the tension-dominant structure depends on both the axial loading and the aspect ratio of length to thickness; $\alpha / \omega_{\mathrm{o}}$ of the curvature-dominant structure depends on the axial loading. The recent experiment by Verbridge et al. [18] shows that the $Q$ factor also depends on the axial loading and very high $Q$ factor is obtained with high tensile axial stress at room temperature. So when analyzing the nanomechanical resonator, $\mu$ in general should be determined/measured case by case for different axial loading scenarios.

\section{Continuous system of nanomechanical resonator with beam structure}

\subsection{Tension-dominant nonlinearity}

For doubly clamped [10], doubly hinged and hingedclamped non-slack beam structures [27] of tension-dominant nonlinearity, their equation of motion is as follows [32]

$m \frac{\partial^{2} w}{\partial t^{2}}+C \frac{\partial w}{\partial t}+E I \frac{\partial^{4} w}{\partial x^{4}}-\frac{\partial}{\partial x}\left[N(x) \frac{\partial w}{\partial x}\right]=f(t)$.

Here $m$ is the mass per unit length of beam; $C$ is the damping coefficient; $E$ is the Young's modulus; $I=b h^{3} / 12$ (b:width and $h$ : thickness) for a rectangular beam, is the moment of inertia. For a nanometer scale beam, the effects such as surface layer, surface tension [33], the strain gradient [34] can stand out, which effectively changes $E$ and $I$. Furthermore, the effect of surface layer can also have impact on the the damping coefficient of $C$ [35], which indicates the energy dissipation of the system. $w=w(x, t)$ is the beam deflection and $f(t)$ is an external excitation. $N(x)$ is the actual axial loading exerted inside the beam, which consists of the following two parts [32]
$N(x)=N_{\mathrm{o}}+\frac{E A_{\mathrm{s}}}{2 L} \int_{0}^{L}\left(\frac{\partial w}{\partial x}\right)^{2} \mathrm{~d} x$.

$N_{\mathrm{o}}$ is the axial loading independent of $x$. The built-in strain [16], fabrication process [18,36], residual stress [37] and surface stress [38] etc. can all contribute to $N_{\mathrm{O}}$. $A_{\mathrm{s}}$ is the cross section area and $L$ is the length of beam. $\frac{E A_{\mathrm{s}}}{2 L} \int_{0}^{L}\left(\frac{\partial w}{\partial x}\right)^{2} \mathrm{~d} x$ is the tension due to (nonlinear) midplane stretching [39]. The beam is modeled as the EulerBernoulli beam which requires the relatively large aspect ratio of length to thickness/radius and the shear effects are thus neglected. Otherwise, Timoshenko beam theory needs to be used [40]. Besides the Euler-Bernoulli beam assumption, three other important assumptions of beam flexural motion are (implicitly) used during the derivation of Eq. (18). The first one is the assumption of no out-of-plane displacement. The coupling of the in-plane and out-of-plane displacements/motions is quite possible in some nanomechanical resonators [16], which may result in "ballooning" motion. For this ballooning motion study, reader should refer to the theoretical and experimental studies done by Narasimha [41], Bayly and Murphy [42]. The second one is to assume the uncoupling of the flexural displacement with axial one. In certain scenarios, the axial inertia effects are the dominant nonlinearity [28] and the coupling effects of flexural and axial displacements are very significant to the system dynamic response $[43,44]$. The uncoupling of flexural and axial displacement can only be valid when these two conditions are satisfied (1): shear and rotary inertia effects are negligible and (2): $u=O\left(w^{2}\right)(u$ is axial displacement and $w$ is flexural one) [27]. Although we assume the Euler-Bernoulli beam model, shear effects are neglected in a sense of static study or beam vibrating with the dominant first mode shape. As nanomechanical resonator can vibrate dominantly with other higher mode shape, shear together with rotary inertia (i. e. coupling effects) can be very significant. Condition (1) can be satisfied only when wavelength of flexural vibration is large compared with the radius of gyration of cross section [27]. Condition (2) is equivalent to say that the radius of gyration is very small, therefore, the axial/longitudinal inertia and damping effects are neglected [27]. These two conditions form the basis for most of the studies of nonlinear beam vibrations [27]. Nanotube and nanowire-based nanomechanical resonator has the large aspect ratio of length to thickness/radius $[4,10]$ and the nonlinearity study in this paper is only for the nanomechanical resonator excited around the fundamental characteristic frequency (eigenfrequency of the first mode shape). Therefore, the uncoupling conditions are satisfied. The third one is that the linear curvature approximation relation of $\kappa=w_{x x}$ is used during the derivation. The exact nonlinear curvature definition is $\kappa=w_{x x} /\left(1+w_{x}^{2}\right)^{3 / 2}$. Curvature $\kappa$ is directly related to the strain of bending beam, and thus system potential energy [36-38]. For the structures of tension-dominant nonlinearity and large aspect ratio, such linear curvature ap- 
proximation is sufficiently accurate [45] and this curvatureinduced nonlinearity in the structures of tension-dominant nonlinearity is also shown negligible in our results. Besides the kinematic assumptions above, there are two physical assumptions are also included in the derivation of Eq. (18). The first is to assume that there is no defect in the resonator or its effect can be ignored. This is reflected in that the bending stiffness EI in Eq. (18) is assumed constant. Defect like crack inside the resonator during the vibration may experience the "breathing" motion, i.e., the crack opens and closes $[46,47]$. The "breathing" crack will cause the change of bending stiffness and thus the dynamic response of the system is affected. The second is that the electrostatic and van der Waals forces between the resonator and the substrate are not included. These two forces are deflection-dependent and nonlinear $[31,48]$, so they can contribute significantly to the system nonlinear dynamic response, which can also cause the pull-in instability [10]. Physically it is no need to consider these two forces' influence when the electrostatic voltage is small and the gap distance between the resonator and the substrate is large.

The following dimensionless quantities are introduced for dimensional analysis

$\tau=\sqrt{\frac{E I}{m L^{4}}}, \quad \xi=\frac{x}{L}, \quad W=\frac{w}{L}$.

Equation (18) is now non-dimensionalized as the following equation

$$
\begin{aligned}
W_{, \tau \tau} & +\frac{C}{m} \sqrt{\frac{m L^{4}}{E I}} W_{, \tau}+W_{, \xi \xi \xi \xi}-\frac{N_{\mathrm{o}} L^{2}}{E I} W_{, \xi \xi} \\
& -\frac{A_{\mathrm{s}} L^{2}}{2 I}\left(\int_{0}^{1} W_{, \xi}^{2} \mathrm{~d} \xi\right) W_{, \xi \xi}=\frac{f(t) L^{3}}{E I} .
\end{aligned}
$$

Here ()$_{, \xi}=\partial / \partial \xi$ and ()$_{, \tau}=\partial / \partial \tau$. We define two dimensionless parameters here, $\Lambda_{1}=N_{\mathrm{o}} L^{2} /(E I)$, which is dimensionless external axial loading and $\Lambda_{2}=A_{\mathrm{s}} L^{2} /(2 I)$. For the beam with rectangular cross section, $A_{\mathrm{s}}=b h$ and $I=b h^{3} / 12 . b$ and $h$ are the beam width and thickness, respectively. Thus, $\Lambda_{2}=6(L / h)^{2}$ and $L / h$ is the beam aspect ratio of length to thickness. Galerkin method is applied for the computation of Eq. (21). First, $W(\xi, \tau)$ is expanded as the following form $[31,49]$

$W(\xi, \tau)=\sum_{i=1}^{\infty} \psi_{i}(\xi) \phi_{i}(\tau)=\psi_{1}(\xi) \phi_{1}(\tau)$,

where $\psi_{i}(\xi)$ is the $i$-th mode shape of beam and $\phi_{i}(\tau)$ is the $i$-th modal amplitude. The $i$-th orthogonal mode shape of doubly hinged beam is given as $\sqrt{2} \sin (\mathrm{i} \pi \xi)$ ( $\sqrt{2}$ is to keep the mode shape normal). And 16 orthonormal mode shapes of doubly clamped beam are given by Chang and Craig [50]. The mode shape of hinged-clamped can be found out by taking the same approach of Ref. [50]. We only study the dynamic steady-state response of the system driven with the frequency around the first eigenfrequency. In Eq. (22), keeping only one mode shape in the expansion is valid and ac- curate because the motions of other mode shapes excited at beginning stage die out with increasing time due to damping [27]. When the driven frequency is around any other (higher) eigenfrequency, the mode uncoupling of keeping only one corresponding mode shape [10] is not valid any more and will lead to serious mistakes [27]. Substitute $W(\xi, \tau)$ of Eq. (22) into Eq. (21) and integrate it from 0 to 1 , Eq. (21) becomes Duffing equation of Eq. (1)

$\psi_{1, \tau \tau}+2 \epsilon \mu_{1} \psi_{1, \tau}+\omega_{1}^{2} \psi_{1}+\epsilon \alpha_{1} \psi_{1}^{3}=\epsilon k_{1} \cos (\Omega t)$,

if we let

$$
\begin{aligned}
& 2 \epsilon \mu_{1}=\frac{C}{m} \sqrt{\frac{m L^{4}}{E I}}, \\
& \omega_{1}^{2}=\frac{\int_{0}^{1} \phi_{1} \phi_{1, \xi \xi \xi \xi} \mathrm{d} \xi-\Lambda_{1} \int_{0}^{1} \phi_{1} \phi_{1, \xi \xi} \mathrm{d} \xi}{\int_{0}^{1} \phi_{1}^{2} \mathrm{~d} \xi}, \\
& \epsilon \alpha_{1}=\frac{-\Lambda_{2} \int_{0}^{1} \phi_{1, \xi}^{2} \mathrm{~d} \xi \int_{0}^{1} \phi_{1} \phi_{1, \xi \xi} \mathrm{d} \xi}{\int_{0}^{1} \phi_{1}^{2} \mathrm{~d} \xi}, \\
& \epsilon k_{1} \cos (\Omega t)=\frac{f(\tau) L^{3}}{E I} \frac{\int_{0}^{1} \phi_{1} \mathrm{~d} \xi}{\int_{0}^{1} \phi_{1}^{2} \mathrm{~d} \xi},
\end{aligned}
$$

where $\omega_{1}$ is the eigenfrequency of the first mode shape and dependent of $\Lambda_{1}$ (dimensionless axial loading). When $\omega_{1}$ drops to zero at critical negative (compressive) $\Lambda_{1}$, it indicates the buckling instability [51].

Now it seems that we can use the same perturbation method introduced above to solve the dynamic response of the continuous system driven around its first eigenfrequency. However, we can not do so at all with Eq. (23), which is non-dimensionalized from Eq. (20). In Duffing equation of Eq. (1), $\epsilon \alpha$ is assumed at least one order magnitude smaller than $\omega_{0}^{2}$, thus we can equate those coefficients of $\epsilon^{0}$ and $\epsilon^{1}$ terms separately to find out the solutions. In Eq. (23), on the contrary, $\epsilon \alpha_{1}$ is much larger than $\omega_{1}^{2}$ if those corresponding mode shape functions of doubly hinged, hinged-clamped and doubly clamped beams are substituted into Eqs. (24) and (25) for the computation, especially when nanomechanical resonator has very large $\Lambda_{2}$. To make the governing equation suitable for the perturbation method to solve, the following dimensionless quantities instead of those in Eq. (20) are introduced [28]

$\tau=\sqrt{\frac{E I Z_{n}^{4}}{m L^{4}}}, \quad \xi=\frac{x Z_{n}}{L}, \quad W=\frac{w Z_{n}}{L}$,

where $Z_{n}$ is a positive dimensionless number, which is related with the $n$-th eigenfrequency $\left(\omega_{n}\right)$ of the system as the following equation $[28,50]$ 
$Z_{n}^{4}=\frac{m L^{4} \omega_{n}^{2}}{E I}$

The corresponding dimensionless governing equation now becomes

$\psi_{1, \tau \tau}+2 \epsilon \mu_{z n} \psi_{1, \tau}+\omega_{z n}^{2} \psi_{1}+\epsilon \alpha_{z n} \psi_{1}^{3}=\epsilon k_{z n} \cos (\Omega t)$,

if we let

$$
\begin{aligned}
& 2 \epsilon \mu_{z n}=\frac{C}{m} \sqrt{\frac{m L^{4}}{E I Z_{n}^{4}}}, \\
& \omega_{z n}^{2}=\frac{\int_{0}^{Z_{n}} \phi_{z n} \phi_{z n, \xi \xi \xi \xi} \mathrm{d} \xi-\frac{\Lambda_{1}}{Z_{n}^{2}} \int_{0}^{Z_{n}} \phi_{z n} \phi_{z n, \xi \xi} \mathrm{d} \xi}{\int_{0}^{1} \phi_{z n}^{2} \mathrm{~d} \xi}, \\
& \epsilon \alpha_{z n}=\frac{-\Lambda_{2} \int_{0}^{Z_{n}} \phi_{z n, \xi}^{2} \mathrm{~d} \xi \int_{0}^{Z_{n}} \phi_{z n} \phi_{z n, \xi \xi} \mathrm{d} \xi}{\int_{0}^{Z_{n}} \phi_{z n}^{2} \mathrm{~d} \xi}, \\
& \epsilon k_{z n} \cos (\Omega t)=\frac{f(\tau) L^{3}}{E I Z_{n}^{3}} \frac{\int_{0}^{Z_{n}} \phi_{z n} \mathrm{~d} \xi}{\int_{0}^{Z_{n}} \phi_{z n}^{2} \mathrm{~d} \xi} .
\end{aligned}
$$

It is noticed that the integral upper limits of Eqs. (29) and (30) are $Z_{n}$. The corresponding orthonormal mode shape is $\phi_{z n}(\xi)=\phi_{1}(\xi) / \sqrt{Z_{n}}[28]$ and $\epsilon \alpha_{z n}=\epsilon \alpha_{1} / Z_{n}^{3}$. Once a proper $Z_{n}$ is chosen to make $\epsilon \alpha_{z n}$ small and suitable for the perturbation method, the exact solution procedures given above for Duffing equation can now be used to solve Eq. (28). While, the interest here is not to solve Eq. (28) as it exactly matches Duffing equation of Eq. (1) and we have already done it in the section above. Although the use of Eq. (26) as the non-dimensionalization scheme makes the governing equation a solvable Duffing equation for perturbation method, it is not proper to use $\epsilon \alpha_{z n} / \omega_{z n}$ to evaluate the nonlinearity for different structures. $Z_{n}$ is different for the beam structure with different boundary conditions. For example, for a doubly hinged beam, $Z_{1}=\pi, Z_{2}=2 \pi, Z_{3}=3 \pi, \cdots$, and $Z_{n}=n \pi$; for a doubly clamped beam, $Z_{n}$ is solved from equation $\cos \left(Z_{n}\right) \cosh \left(Z_{n}\right)=1$, and $Z_{1}=4.73, Z_{2}=7.85$, $Z_{3}=10.995$ [50]. Therefore, the non-dimensionalization scheme of Eq. (26) varies for the structures with the same given material elastic properties and structure dimensions but different boundary conditions. So if $\epsilon \alpha_{z n} / \omega_{z n}$ is used to evaluate the nonlinearity of different structures, it is not an apple-to-apple comparison. Instead of $\epsilon \alpha_{z n} / \omega_{z n}$, we use $\epsilon \alpha_{1} / \omega_{1}$ to evaluate the nonlinearity of different structures. We are fully aware that $\epsilon \alpha_{1} / \omega_{1}$ is a large number due to the non-dimensionalization scheme of Eq. (20). $\frac{\epsilon \alpha_{z n}}{\omega_{z n}}=\frac{1}{Z_{n}^{3}} \frac{\epsilon \alpha_{1}}{\omega_{1}}$ when $\Lambda_{1}=0$ and $\frac{\epsilon \alpha_{z n}}{\omega_{z n}} \propto \frac{1}{Z_{n}^{3}} \frac{\epsilon \alpha_{1}}{\omega_{1}}$ for a moderate $\Lambda_{1}$. This $\epsilon \alpha_{1} / \omega_{1}$ parameter has already been used by Postma et al. in their study on the critical amplitude for the onset of nonlinearity as shown in their Eq. (5) [10], which is the following

$a_{\mathrm{c}}=\omega_{1} \frac{L^{2}}{\pi^{2}} \sqrt{\frac{\rho \sqrt{3}}{E Q}}=\frac{4}{3} \sqrt{\sqrt{3} \epsilon \mu\left(\frac{\epsilon \alpha_{1}}{\omega_{1}}\right)^{-1}}$,

with corresponding $2 \epsilon \mu=\omega_{1} / Q$ and $\epsilon \alpha_{1}=\frac{E}{18 \rho}\left(\frac{2 \pi}{L}\right)^{4}$. Here $a_{\mathrm{c}}$ is the critical amplitude; $Q$ is the quality factor and $\rho$ is the density. For a softening Duffing oscillator with negative $\epsilon \alpha_{1} / \omega_{1}, \epsilon \alpha_{1} / \omega_{1}$ above is substituted by $-\epsilon \alpha_{1} / \omega_{1}$.

\subsection{Curvature-dominant nonlinearity}

Instead of $\kappa=w_{x x}$, the exact nonlinear curvature definition of $\kappa=w_{x x} /\left(1+w_{x}^{2}\right)^{3 / 2}$ is used for the derivation of cantilever beam of curvature-dominant nonlinearity. By using the nonlinear curvature and Hamilton's principle, the following governing equation of cantilever beam is derived, in which the higher (than cubic) order terms have already been truncated

$$
\begin{aligned}
m \frac{\partial^{2} w}{\partial t^{2}}+ & C \frac{\partial w}{\partial t}+E I \frac{\partial^{4} w}{\partial x^{4}}-N_{\mathrm{o}} \frac{\partial^{2} w}{\partial x^{2}} \\
& +E I \frac{\partial}{\partial x}\left[\frac{\partial w}{\partial x} \frac{\partial}{\partial x}\left(\frac{\partial w}{\partial x} \frac{\partial^{2} w}{\partial x^{2}}\right)\right]=f(t) .
\end{aligned}
$$

Compared with Eq. (18), the nonlinear term due to midplane stretching is gone; compared with the model of Anderson et al. [28], the axial loading $N_{\mathrm{o}}$ is incorporated. In the structures of tension-dominant, the ends are either hinged or clamped, which prevents the beam span from extending horizontally. Only under this condition, the tension due to mid-plane stretching can exist. For cantilever beam, one end is free, therefore, there is no tension due to mid-plane stretching in the governing equation. With the same nondimensionalization scheme and modal discretization given in Eqs. (20) and (22), the same dimensionless form of governing equation as Eq. (23) is derived. The only difference is the definition of $\epsilon \alpha_{1}$. For cantilever beam of curvature-dominant nonlinearity, $\epsilon \alpha_{1}$ is defined as follows

$\epsilon \alpha_{1}=\frac{\int_{0}^{1} \phi_{1}\left[\phi_{1, \xi \xi}^{3}+4 \phi_{1, \xi} \phi_{1, \xi \xi} \phi_{1, \xi \xi \xi}+\phi_{1, \xi}^{2} \phi_{1, \xi \xi \xi \xi}\right] \mathrm{d} \xi}{\int_{0}^{1} \phi_{1}^{2} \mathrm{~d} \xi}$.

An very important $\epsilon \alpha_{1}$ difference of curvature-dominant nonlinearity with that of tension-dominant nonlinearity is that $\epsilon \alpha_{1}$ of curvature-dominant nonlinearity is independent of $\Lambda_{2}$ (aspect ratio). This leads to the following conclusion: tension-dominant nonlinearity of $\epsilon \alpha_{1} / \omega_{1}$ depends on both $\Lambda_{1}$ and $\Lambda_{2}$; curvature-dominant nonlinearity of $\epsilon \alpha_{1} / \omega_{1}$ depends on $\Lambda_{1}$ only.

The mode shapes of a cantilever beam given by Chang and Craig [50] are for the cantilever beam of no axial loading case. Axial loading appears only in the governing equations of the structures of tension-dominant nonlinearity, such 
as doubly hinged, hinged-clamped and clamped-clamped beams. Unlike those structures of tension-dominant nonlinearity, axial loading appears in both governing equation and boundary conditions of cantilever beam (see Appendix and also Ref. [51]). Due to that the boundary conditions of cantilever beam vary with axial loading, the mode shapes given by Chang and Craig can not be used in Galerkin method for the case of cantilever beam with an axial loading because those mode shapes do not satisfy the boundary conditions. The derivation of mode shapes of a cantilever beam is given in Appendix.

\section{Results and discussion}

For single DOF system, the system effective stiffness information is incorporated in parameters of $\omega_{0}^{2}, \omega_{1}^{2}$ in Eqs. (1) and (23). Higher resonant frequency means higher effective stiffness with given mass per unit length. Buckling load is another measure. Stiffer structure requires larger magnitude of (compressive) buckling load. When $\omega_{1}=0$, buckling happens, therefore, the dimensionless buckling $\Lambda_{1}$ is derived from Eq. (24) as the following equation

$\Lambda_{1}^{\mathrm{c}}=\frac{\int_{0}^{1} \phi_{1} \phi_{1, \xi \xi \xi \xi} \mathrm{d} \xi}{\int_{0}^{1} \phi_{1} \phi_{1, \xi \xi} \mathrm{d} \xi}$.

$\Lambda_{1}^{\mathrm{c}}=-\pi^{2}$ for doubly hinged beam. $\Lambda_{1}^{\mathrm{c}} \approx-4 \pi^{2},-(\pi / 0.7)^{2}$, and $-\pi^{2} / 4$ for doubly clamped, clamped-hinged and cantilever beams, respectively [51]. Figure 5 shows $\omega_{1}$ variation with $\Lambda_{1}$. As shown in Fig. 5, the doubly clamped beam is the stiffest and the cantilever is the most flexible among these four structures. It is worth mentioning again that $\omega_{1}$ is the eigenfrequency of the first mode, which is derived from

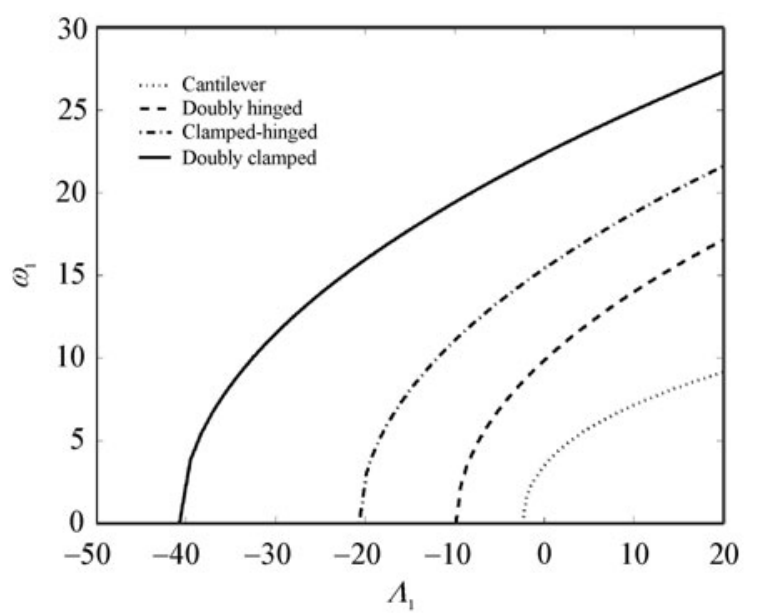

Fig. 5 The fundamental frequencies of four beam structures as the functions of axial loading. For each beam structure, the axial loading starts from its buckling load
Eq. (24) and contains linear part frequency information only. $\omega_{1}=0$ does not mean that the system frequency of Eq. (23) is zero because the nonlinear term also contributes to the system stiffness. When $\Lambda_{1} \leq \Lambda_{1}^{\mathrm{c}}$, statically beam will experience a postbuckling deformation of pitchfork bifurcation [52]. Static part (when all time-related terms are eliminated) of Eq. (23) is capable of describing the postbuckling behavior of the system and Eq. (23) can also be used to study the dynamic response of buckled beam. However, in buckling/postbuckling region, the system will have chaotic motion of two-well potential attractor due to the pitchfork bifurcation [53]. When tuning nanomechanical resonator, axial loading should not be less than the critical buckling loading because the initial-condition-sensitive chaotic response make it very difficult to control the resonator response if not impossible.

Figures 6-8 show the $\epsilon \alpha_{1} / \omega_{1}$ variation with $\Lambda_{1}$ and $\Lambda_{2}$ for the tension-dominant structures of doubly hinged, clamped-hinged and doubly clamped beams, respectively. Because $\Lambda_{1}=\Lambda_{1}^{\mathrm{c}}, \epsilon \alpha_{1} / \omega_{1}=\infty$. We start $\Lambda_{1}$ from $\Lambda_{1}=0.9 \Lambda_{1}^{\mathrm{c}}$ for each beam structure. $\Lambda_{2}$ starts from 300 . The reason is that $\Lambda_{2}=6(L / h)^{2}$ and rule of thumb states Timoshenko beam theory rather than Euler-Bernoulli one should be applied if $L / h<7$. For all these three structures of tension-dominant nonlinearity, $\epsilon \alpha_{1} / \omega_{1}$ is positive, which means hardening effects. All $\epsilon \alpha_{1} / \omega_{1} \mathrm{~s}$ decrease monotonically with increasing $\Lambda_{1}$ and increase monotonically with increasing $\Lambda_{2} . \epsilon \alpha_{1} / \omega_{1}$ of doubly hinged beam is smaller than that of either doubly clamped or clamped-hinged beam for any given given axial loading $\Lambda_{1}$ and $\Lambda_{2}$. It is interesting and important to point out that $\epsilon \alpha_{1} / \omega_{1}$ of a clamped-hinged beam is larger than that of doubly clamped beam though the clamped-hinged beam has lower eigenfrequency. The geometric asymmetry of clamped-hinged beam is responsible for that. And this geometric asymmetry can be used to enhance the system nonlinearity with the sacrifice of higher eigenfrequency compared with that of a doubly clamped beam.

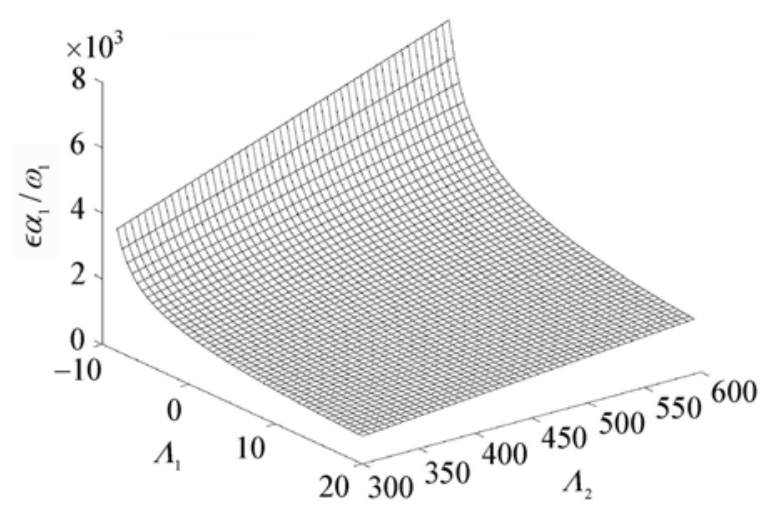

Fig. 6 The nonlinearity coefficient $\epsilon \alpha_{1} / \omega_{1}$ of a doubly hinged beam as a function of $\Lambda_{1}$ and $\Lambda_{2} . \Lambda_{1}$ is dimensionless axial loading and $\Lambda_{2}=6(L / h)^{2} . L$ and $h$ are the beam length and thickness, respectively 


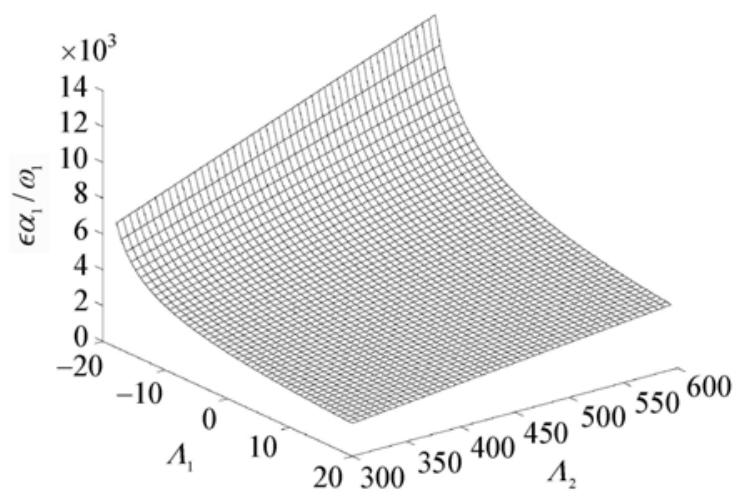

Fig. 7 The nonlinearity coefficient $\epsilon \alpha_{1} / \omega_{1}$ of a clamped-hinged beam as a function of $\Lambda_{1}$ and $\Lambda_{2}$

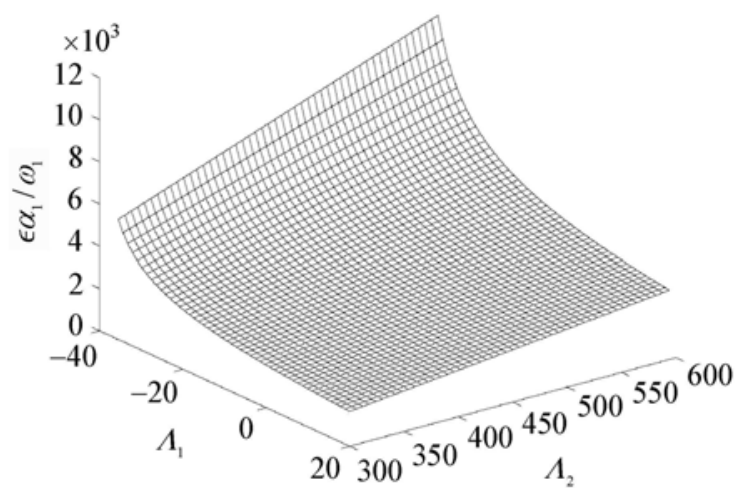

Fig. 8 The nonlinearity coefficient $\epsilon \alpha_{1} / \omega_{1}$ of a doubly clamped beam as a function of $\Lambda_{1}$ and $\Lambda_{2}$

Figure 9 shows $\epsilon \alpha_{1} / \omega_{1}$ variation of a cantilever beam with $\Lambda_{1}$. The analysis above shows that $\epsilon \alpha_{1} / \omega_{1}$ variation is only dependent on $\Lambda_{1} . \epsilon \alpha_{1} / \omega_{1}$ of a cantilever beam monotonically increase with increasing $\Lambda_{1}$, which is opposite compared with those of the structures with tension-dominant nonlinearity. The magnitude of $\epsilon \alpha_{1} / \omega_{1}$ of a cantilever beam is also significantly smaller. Together with Eq. (31), this explains the experimental observation that much larger driving amplitude is needed for critical $a_{\mathrm{c}}$ of cantilevered nanomechanical resonator than that of a doubly clamped beam [10]. It is observed that $\epsilon \alpha_{1} / \omega_{1}$ of a cantilever beam does not always keep positive. In the region of $\Lambda_{1}^{\mathrm{c}}<\Lambda_{1}<-0.929$, $\Lambda_{1}$ is negative, i.e. softening effect and the softening effects are also observed in the nanomechanical memory [5]. For a cantilever beam, $\Lambda_{1}^{\mathrm{c}} \approx \pi^{2} / 4$ and in Fig. 9, $\Lambda_{1}$ also starts from $0.9 \Lambda_{1}^{\mathrm{c}}$. When $\Lambda_{1}>-0.929, \epsilon \alpha_{1} / \omega_{1}$ in Fig. 9 is positive. This implies that when tuning $\Lambda_{1}$ around -0.929 , the dynamic nonlinearity can be eliminated or greatly reduced. For a cantilever beam of curvature-dominant nonlinearity, the geometric asymmetry and interactions of mode shape and its first, second, third and fourth derivatives as indicated in Eqs. (24) and (25) determine the properties of $\epsilon \alpha_{1} / \omega_{1}$.

Once again, reader should be cautious and aware that the present analysis is only to study the dynamic nonlinear response when the driving frequency is around the first eigenfrequency. If the driving frequency is (much) higher, for example, around the second eigenfrequency, the above modeling and results are in general not valid. One assumption used in the derivation is the uncoupling of transverse and axial displacements. In reality, the structure vibrates not only transversely but also axially/longitudinally. The axial/longitudinal (nonlinear) inertia effect can become dominant when driving frequency is around the second eigenfrequency [28]. Equations (18) and (32) of the beam transverse vibration do not incorporate such effect. Both the analysis presented here and theoretical/experimental study by Anderson et al. [28] conclude that a cantilever beam driven around its first eigenfrequency is a hardening Duffing oscillator when $\Lambda_{1}=0$. The hardening effect is due to the fact that the curvature is the dominant factor for the nonlinearity, which is theoretically and experimentally verified and justified [28]. However, the nonlinearity due to axial/longitudinal inertia effects becomes dominant when a cantilever is driven around its second eigenfrequency, which exhibits softening effects [28]. This kind of nonlinear response can help to explain why the errors steadily grow with a dramatic improvement of mass sensitivity in the experiment of cantilever based mass sensor as shown in Ref. [7] when a cantilever is driven around the first, second, third and fourth eigenfrequencies. Dohn et al. modeled the cantilever mass sensor as a linear oscillator [7]. When the system is driven around higher eigenfrequency, the coupling effect of the axial and transverse displacements becomes more and more important because the wavelength of higher mode shape becomes shorter [27]. The linear oscillator model is thus expected to deviate more and more from the experimental data. The nonlinear axial inertia effects (together with other possible nonlinearities) should be in general considered when a cantilever is driven around a higher eigenfrequency for mass sensing. Equation (32) can not predict such softening effects of axial inertia-induced nonlinearity because it does not include the axial nonlinear inertia term in the governing equation.

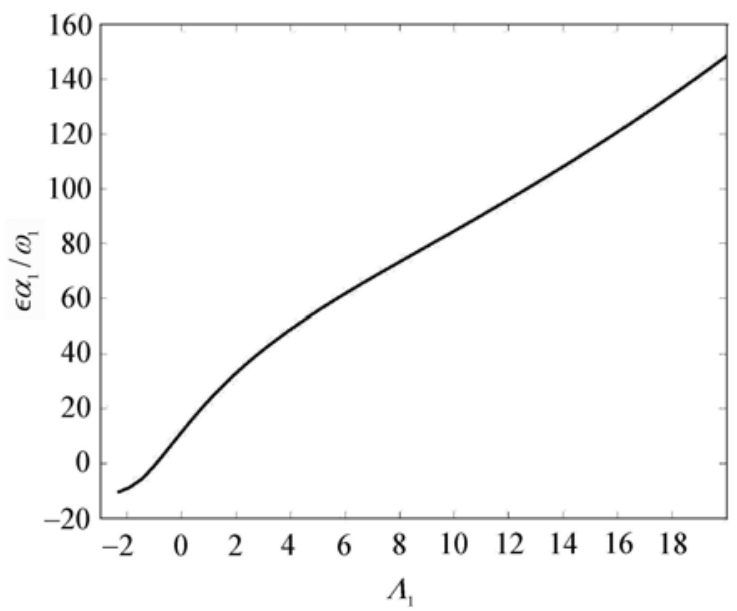

Fig. 9 The nonlinearity coefficient $\epsilon \alpha_{1} / \omega_{1}$ of a cantilever beam as a function of $\Lambda_{1}$ 


\section{Summary}

In conclusion, a new parameter is proposed to systematically evaluate the nonlinearity of the beam dynamic response. Two categories of nonlinearity: tension-dominant and curvature-dominant nonlinearities, are analyzed as a function of axial loading and aspect ratio. In essence, this nonlinearity study is to use modal analysis and Galerkin method as a discretization approach to transform the differential-integral and differential governing equations of continuous system into single DOF Duffing equation. The discussion on the assumptions/limitations of modeling and transforming the governing equation into Duffing equation consists of a very important part in this paper. The applicability range of the modeling and analysis is also addressed during the discussion.

\section{Appendix}

The linear dynamic governing equation for a beam with no damping is

$E I \frac{\mathrm{d}^{4} y}{\mathrm{~d} x^{4}}-p \frac{\mathrm{d}^{2} y}{\mathrm{~d} x^{2}}+m \frac{\mathrm{d}^{2} y}{\mathrm{~d} t^{2}}=0$,

where $y$ is the beam transverse displacement, $m$ is the mass per unit length of the beam, $p$ is the axial load. When $p>0$, it is tensile; when $p<0$, it is compressive.

The beam length is $L$. Assume that $y$ has such solution form, $y=V(x) \mathrm{e}^{\mathrm{i} \omega t}$. Substitute this solution form into Eq. (A1) and introduce dimensional parameter $\xi=x / L$. The governing equation (A1) becomes

$-m \omega^{2} l^{4} V+E I V^{\prime \prime \prime \prime}-p L^{2} V^{\prime \prime}=0$.

Here ()$^{\prime}=\mathrm{d} / \mathrm{d} \xi$. Let $k^{2}=\left\|p L^{2} /(E I)\right\|$ and $\beta^{4}=m \omega^{2} L^{4} /(E I)$. For the tension case of $p>0$, the governing equation is

$V^{\prime \prime \prime \prime}-k^{2} V^{\prime \prime}-\beta^{4} V=0$.

For the compression case of $p<0$, the governing equation is

$V^{\prime \prime \prime \prime}+k^{2} V^{\prime \prime}-\beta^{4} V=0$.

For the tension case, the solution form of $V$ is

$V=A \mathrm{e}^{f_{2} \xi}+B \mathrm{e}^{-f_{2} \xi}+C \sin \left(f_{1} \xi\right)+D \cos \left(f_{1} \xi\right)$.

$A, B, C, D$ are the four unknown constants. $f_{1}$ and $f_{2}$ are defined as $f_{1}=\sqrt{\beta_{1}-k^{2} / 2}$ and $f_{2}=\sqrt{\beta_{1}+k^{2} / 2}$. Here $\beta_{1}=\sqrt{\beta^{4}+k^{4} / 4}$. The four dimensionless boundary conditions are given as

$$
\begin{array}{ll}
V(0)=0, & \frac{\mathrm{d} V(0)}{\mathrm{d} \xi}=0, \\
\frac{\mathrm{d}^{2} V(1)}{\mathrm{d} \xi^{2}}=0, & \frac{\mathrm{d}^{3} V(1)}{\mathrm{d} \xi^{3}}-k^{2} \frac{\mathrm{d} V(1)}{\mathrm{d} \xi}=0 .
\end{array}
$$

Substitute the $V$ solution form into these four boundary conditions, it forms a $4 \times 4$ matrix. Setting the matrix determinant to zero gives the eigenfrequencies of the systems; and then by substituting each individual eigenfrequency into that $4 \times 4$ matrix, the mode shape can be found. For the compression case, the solution has the following form
$V=A \mathrm{e}^{f_{1} \xi}+B \mathrm{e}^{-f_{1} \xi}+C \sin \left(f_{2} \xi\right)+D \cos \left(f_{2} \xi\right)$,

and the boundary conditions change to

$$
\begin{array}{ll}
V(0)=0, & \frac{\mathrm{d} V(0)}{\mathrm{d} \xi}=0, \\
\frac{\mathrm{d}^{2} V(1)}{\mathrm{d} \xi^{2}}=0, & \frac{\mathrm{d}^{3} V(1)}{\mathrm{d} \xi^{3}}+k^{2} \frac{\mathrm{d} V(1)}{\mathrm{d} \xi}=0 .
\end{array}
$$

Repeat the same procedures of tension case to find out eigenfrequencies and mode shapes for the compression case.

\section{References}

1 Cleland, A.N., Roukes, M.L.: Fabrication of high frequency nanometer scale resonators from bulk Si crystals. Appl. Phys. Lett. 69(18), 2653-2655 (1996)

2 Cleland, A.N., Roukes, M.L.: A nanometre-scale mechanical electrometer. Nature 392(6672), 160-162 (1998)

3 Turner, K.L., Miller, S.A., Hartwell, P.G., et al.: Five parametric resonances in a microelectromechanical system. Nature 396(6707), 149-152 (1998)

4 Sazonova, V., Yalsh, Y., Üstünel, H., et al.: A tunable carbon nanotube electromechanical oscillator. Nature 431(7016), 284-287 (2004)

5 Badzey, R.L., Zolfagharkhani, G., Gaidarzhy, A., et al.: A controllable nanomechanical memory element. Appl. Phys. Lett. 85(16), 3587-3589 (2004)

6 Badzey, R.L., Zolfagharkhani, G., Gaidarzhy, A., et al.: Temperature dependence of a nanomechanical switch. Appl. Phys. Lett. 86(2), 023106 (2005)

7 Dohn, S., Sandberg, R., Svendsen, W., et al.: Enhanced functionality of cantilever based mass sensors using higher modes. Appl. Phys. Lett. 86(23), 233501 (2005)

8 Erbe, A., Krömmer, H., Kraus, A., et al.: Mechanical mixing in nonlinear nanomechanical resonators. Appl. Phys. Lett. 77(19), 3102-3104 (2000)

9 Huang, X.M.H., Zorman, C.A., Mehregany, M., et al.: Nanodevice motion at microwave frequency. Nature 421(6931), 496 (2003)

10 Postma, H.W.Ch., Kozinsky, I., Husain, A., et al.: Dynamic range of nanotube- and nanowire-based electromechanical systems. Appl. Phys. Lett. 86(22), 223105 (2005)

11 Gaidarzhy, A., Zolfagharkhani, G., Badzey, R.L., et al.: Evidence for quantized displacement in macroscopic nanomechanical oscillators. Phys. Rev. Lett. 94(3), 030402 (2005)

12 Ekinci, K.L., Huang, X.M.H., Roukes, M.L.: Ultrasensitive nanoelectromechanical mass detection. Appl. Phys. Lett. 84(22), 4469-4471 (2004)

13 Evoy, S., Carr, D.W., Sekaric, L., et al.: Nanofabrcaition and electrostatic operation of single-crystal silicon paddle oscillators. J. Appl. Phys. 86(11), 6072-6077 (1999)

14 Papadakis, S.J., Hall, A.R., Williams, P.A., et al.: Resonant oscillators with carbon-nanotude tosion springs. Phys. Rev. Lett. 93(14), 146101 (2004)

15 Craighead, H.G.: Nanomechanical systems. Science 290(5496), 1532-1535 (2000) 
16 Kozinsky, I., Postma, H.W.Ch., Bargatin, I., et al.: Tuning nonlinearity, dynamic range, and frequency of nanomechanical resonators. Appl. Phys. Lett. 88(25), 253101 (2006)

17 Ekinci, K.L., Roukes, M.L.: Nannomechanical systems. Rev. Sci. Instrum. 76(6), 061101 (2005)

18 Verbridge, S.S., Parpia, J.M., Reichenbach, R.B., et al.: High quality factor resonance at room temperature with nanostrings under high tensile stress. J. Appl. Phys. 99(12), 124304 (2006)

19 Roukes, M.L.: Nanoelectromechanical systems face the future. Phys. World 14, 25-31 (2001)

20 Cho, A.: Researchers race to put the quantum into mechanics. Science 299(5603), 36-37 (2003)

21 Mohanty, P., Harrington, D.A., Ekinci, K.L., et al.: Intrinsic dissipation in high-frequency micromechanical resonators. Phys. Rev. B 66(8), 085416 (2002)

22 Knobel, R., Cleland, N.A.: Nanometre-scale displacement sensing using a single electron transistor. Nature 424(6946), 291-293 (2003)

23 Peano, V., Thorwart, M.: Macroscopic quantum effects in a strongly driven nanomechanical resonator. Phys. Rev. B 70(23), 235401 (2004)

24 Dykman, M.I., Fistul, M.V.: Multiphoton antiresonance. Phys. Rev. B 71(14), 140508 (R) (2005)

25 Krömmer, H., Erbe, A., Tilke, A., et al.: Nanomechanical resonators operating as charge detectors in the nonlinear regime. Europhys. Lett. 50(1), 101-106 (2000)

26 Greywall, D.S., Yurke, B., Busch, P.A., et al.: Evading amplifier noise in nonlinear oscillation. Phys. Rev. Lett. 72(19), 2992-2995 (1994)

27 Nayfeh, A.H., Mook, D.T.: Nonlinear Oscillation, John Wiley \& Sons, Inc., New York, 1979

28 Anderson, T.J., Nayfeh, A.H., Balachandran, B.: Experimental verification of the importance of the nonlinear curvature in the response of a cantilever beam. J. of Vibr. Acoust. 118(1), 21-27 (1996)

29 Cleland, A.N.: Carbon nanotubes tune up. Nature 431(7006), 251 (2004)

30 Lifshitz, R., Cross, M.T.: Nonlinear dynamics of nanomechanical and micromechanical resonators. In: Schuster, H.G. ed. Reviews of Nonlinear Dynamics and Complexity, Wiley-VcH Verlag GmbH \& Co. KGaA, Weinbeim, Germany, 2009

31 Zhang, Y., Zhao, Y.P.: Numerical and analytical study on the pull-in instability of micro-structure under electrostatic loading. Sensors and Actuators A 127(2) 366-380 (2006)

32 Tseng, W.Y., Dugundji, J.: Nonlinear vibrations of a beam under harmonic excitation. J. Appl. Mech, 37(2), 292-297 (1970)

33 Guo, J.G., Zhao, Y.P.: The size-dependent bending elastic properties of nanobeams with surface effects. Nanotechnology, 18, 295701 (2007)

34 Ji, B., Chen, W., Zhao, J.: Measurement of length-scale and solution of cantilever beam in couple stress elasto-plasticity. Acta. Mech. Sin., 25(3), 381-387 (2009)
35 Hasheminejad, S.M., Gheshlaghi, B.: Dissipative surface effects on free vibrations of nanowires. Appl. Phys. Lett. 97(25), 253103 (2010)

36 Zhang, Y.: Deflections and curvatures of a film-substrate with the presence of gradient stress in MEMS applications. J. Micomech. Micoeng. 17, 753-762 (2007)

37 Zhang, Y., Zhao, Y.P.: Applicability range of Stoney's formula and modified formulas for a film/substrate bilayer. J. Appl. Phys. 99(5), 053513 (2006)

38 Zhang, Y., Ren, Q., Zhao, Y.P.: Modelling analysis of surface stress on a rectangular cantilever beam. J. Phys. D: Appl. Phys. 37, 2140-2145 (2004)

39 McDonald Jr., P.H., Raleigh, N.C.: Nonlinear dynamic coupling in a beam vibration. J. Appl. Mech. 22(4), 573-578 (1955)

40 Herrmann, G.: Forced motions of Timoshenko beams. J. Appl. Mech. 22(1), 53-56 (1955)

41 Narasimha, R.: Non-linear vibration of an elastic string. J. Sound Vib. 8(1), 134-146 (1968)

42 Bayly, P.V., Murphy, K.D.: Coupling between dissimilar modes in an asymmetrically forced string. J. Acoust. Soc. Am. 103(6), 3362-3369 (1998)

43 Evensen, H.A., Evan-Iwanowski, R.M.: Effects of longitudinal inertia upon the parametric response of elastic column. J. Appl. Mech. 33(1), 141-148 (1966)

44 McIvor, I.K., Bernard, J.E.: The dynamic response of columns under short duration axial loads. J. Appl. Mech. 40(3), 688692 (1973)

45 Thurman, A.L., Mote, C.D.: Free, periodic, nonlinear oscillation of an axially moving strip. J. Appl. Mech. 36(1), 83-91 (1969)

46 Pugno, N., Surface, C., Ruotolo, R.: Evaluation of the nonlinear dynamic response to harmonic excitation of a beam with several breathing cracks. J. Sound Vib. 235(5), 749-762 (2000)

47 Murphy, K.D., Zhang, Y.: Vibration and stability of a cracked translating beam. J. Sound Vib. 237(2), 319-335 (2000)

48 Ke, C.H., Pugno, N., Peng, B., et al.: Experiments and modeling of carbon nanotube-based NEMS devices. J. Mech. Phys. Solids 53(6), 1314-1333 (2005)

49 Feng, Z., Hu, H.: Principal parametric and three-to-one internal resonances of flexible beams undergoing a large linear motion. Acta Mech. Sin., 19(4), 355-364 (2003)

50 Chang, T.C., Craig Jr., R.R.: Normal modes of uniform beams. J. Eng. Mech. Div. ASCE 95(EM4), 1027-1031 (1969)

51 Chajes, A.: Principles of Structural Stability Theory. PrenticeHall, Inc., Englewood Cliffs, NJ, 1974

52 Thompson, J.M.T., Hunt, G.W.: Elastic Instability Phenomena. Jonh Wiley \& Sons, Inc., New York, 1984

53 Moon, F.C.: Chaotic and Fractal Dynamics: An Introduction to Applied Scientists and Engineers. Jonh Wiley \& Sons, Inc., New York, 1992 